\title{
Duplex ultrasound for evaluation of deep venous blood flow in fractured lower extremities
}

\author{
Awadalla Adam ${ }^{1 A, B, C, D, E, F}$, , Mohamed Yousef ${ }^{1,2 A, B, C, D, E, F}$, Babiker A. Wahab ${ }^{1 A, B, C, D, E, F}$, Ahmed Abukonna ${ }^{1 A, B, C, D, E, F}$, \\ Mustafa Z. Mahmoud ${ }^{3 A, B, C, D, E, F}$ \\ 'Department of Diagnostic Radiologic Technology, College of Medical Radiological Sciences, Sudan University of Science and Technology, Khartoum, Sudan \\ ${ }^{2}$ Radiologic Sciences Program, Batterjee Medical College, Jeddah, Saudi Arabia \\ ${ }^{3}$ Radiology and Medical Imaging Department, College of Applied Medical Sciences, Prince Sattam bin Abdulaziz University, Al-Kharj, Saudi Arabia
}

\section{Abstract}

Purpose: Early identification of deep venous thrombosis (DVT) in trauma patients would result in an early initiation of treatment, thereby decreasing the frequency of complications. The aim of the current study was to evaluate the role of duplex ultrasound (DUS) in the evaluation of deep venous blood flow in fractured lower extremities to rule out DVT prior to orthopedic surgery.

Material and methods: In this prospective study a total of 58 patients ( 42 males and 16 females; mean age of $51.5 \pm$ 19.5 years) with fractured lower extremities were thoroughly evaluated prior to surgery with respect to medical history, fracture pattern, associated injuries, comorbid conditions, and venous duplex ultrasound (VDUS) findings. Each affected limb was assessed for the presence of DVT using a Sonoline G 60S ultrasound unit. The analysis was performed with the Statistical Package for the Social Sciences (SPSS) version 20.

Results: DVT was found in $36(62.1 \%)$ patients with single closed fractures, 9 (15.5\%) patients with single opened fractures, 10 (17.2\%) patients with multiple closed fractures, and in $3(5.2 \%)$ patients with multiple opened fractures. Sensitivity and specificity of the findings of compressibility and phasicity for DVT detection in patients with fractured lower extremities were $81.25 \%$ and $87.50 \%$ and $100 \%$ and $100 \%$ respectively. In addition, the absence of compressibility and phasicity had positive predictive value of $100 \%$ and $100 \%$ and negative predictive value of $93.75 \%$ and $95.65 \%$ respectively.

Conclusions: US of DVs in the brightness mode (B-mode) with compression maneuvers should be the first-line imaging modality for suspected DVT in patients with fractured lower extremities.

Key words: ultrasonography, Doppler, duplex, venous thromboembolism, venous thrombosis.

\section{Introduction}

Deep venous thrombosis (DVT) is a common disease with potentially serious consequences such as pulmonary embolism (PE). It should be noted that venous thromboembolism (VTE) includes both DVT and PE [1,2]. The incidence of DVT in the general population is between
1.6 and 1.8 per 1.000 per year [1]. Three factors, namely, blood flow, hypercoagulability, and endothelial injury play and important role in the process of thrombus formation and they are referred to as Virchow's triad. Notably, major trauma often precipitates one or all of these risk factors [3]. Trauma patients often have all three of these factors, which causes a high risk of thromboembolism. Direct injury to

\section{Correspondence address:}

Mustafa Z. Mahmoud, Radiology and Medical Imaging Department, College of Applied Medical Sciences, Prince Sattam bin Abdulaziz University, P0 Box 422,

Al-Kharj 11942, Saudi Arabia, e-mail: m.alhassen@psau.edu.sa

Authors' contribution:

A Study design · B Data collection · C Statistical analysis · D Data interpretation · E Manuscript preparation · F Literature search · G Funds collection 


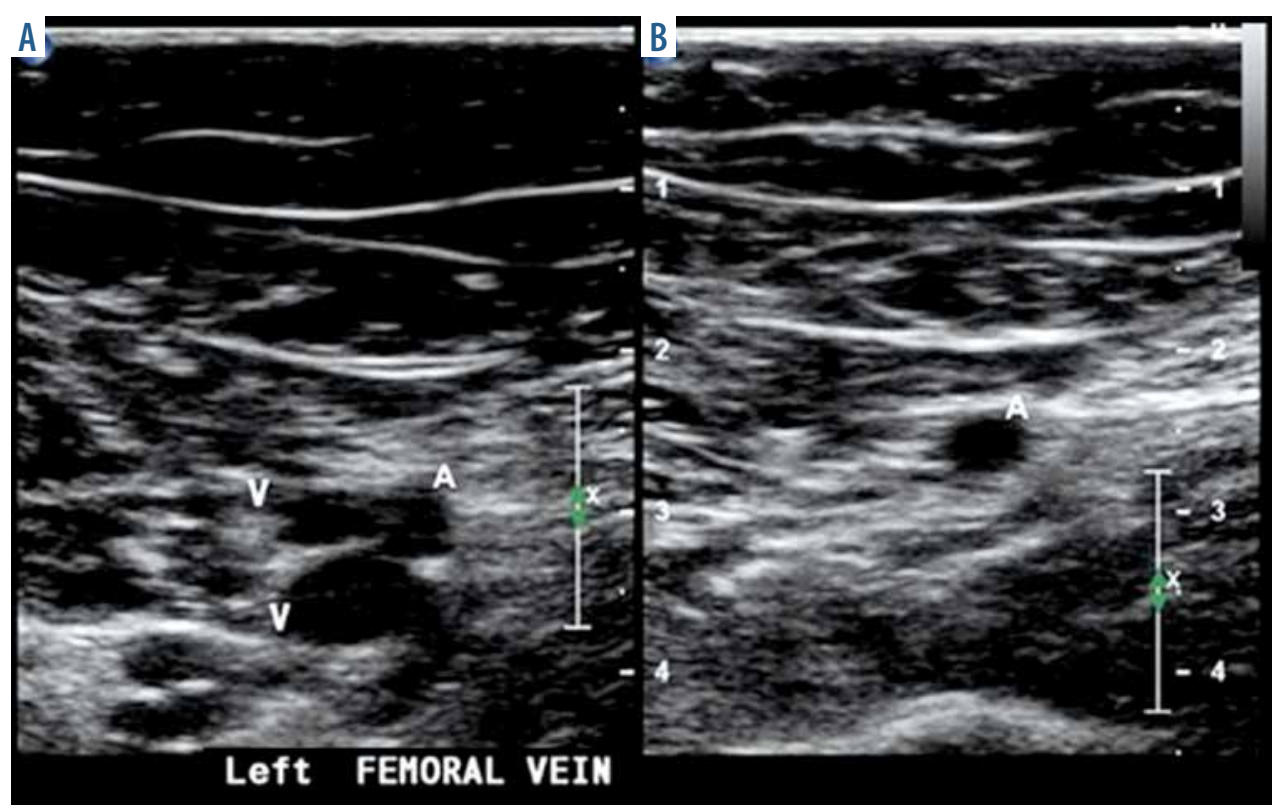

Figure 1. Normal gray-scale ultrasound exam of the veins with a compression scheme. A) Duplicating femoral veins ' $V$ ' next to superficial femoral artery 'A'. B) Both femoral veins are compressed fully by pressure exerted by a ultrasound probe [9]

blood vessels can cause intimal damage, leading to thrombosis, prolonged bed rest, immobilization, hypoperfusion, and paralysis, all promoting venous stasis [4]. The association between injury and VTE is well recognized, and the reported incidence of VTE after trauma varies from $7 \%$ to $58 \%$ depending on patient demographics, kind of injury, method of detection, and type of VTE prophylaxis used [5]. Vascular injuries constitute less than 3\% of all traumatic findings, yet they are associated with potentially serious events. Injuries of the greater blood vessels may have devastating complications resulting in amputations or even death. An understanding of the mechanism of trauma is of utmost importance for accurate diagnosis [6,7].

The majority of vessel injuries affect the upper and lower limbs. Vessel injury is a serious problem for phy-

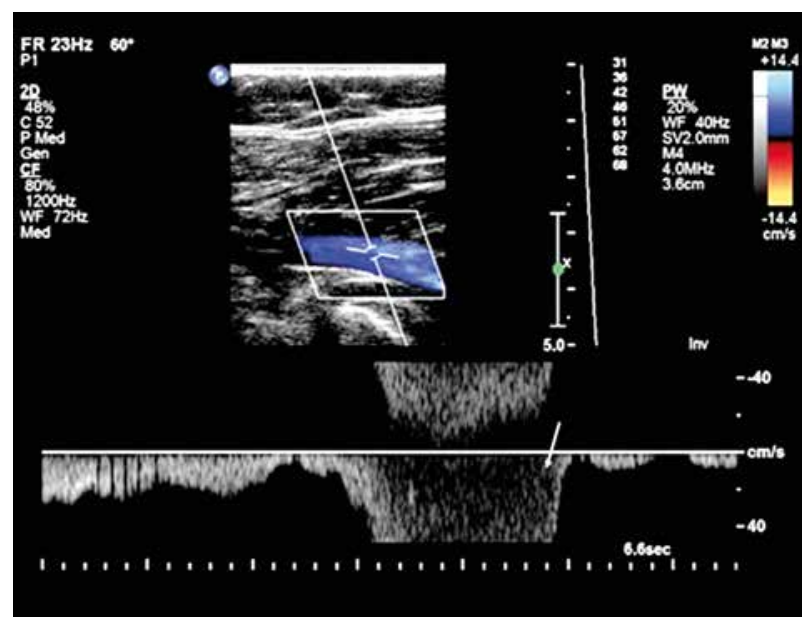

Figure 2. Spectral Doppler waveform analysis of the lower limb veins. Spontaneous and respirophasic flow with a typical response to an augmentation maneuver and aliasing of the pulsed Doppler waveform (arrow) [9] sicians, as some vascular lesions may not be initially recognized based on clinical evaluation and vital signs. Thus, imaging and follow-up should be the basis for diagnosis and care [6]. Angiography is the gold standard method for the examination of trauma-related vascular complications; however, noninvasive diagnostic modalities such as computed tomography angiography (CTA), magnetic resonance angiography (MRA), and venous duplex ultrasound (VDUS) have recently emerged as adequate alternatives [8]. VDUS uses two components to assess for DVT, namely, the brightness mode (B-mode) imaging with probe compression and Doppler evaluation including color flow Doppler imaging and spectral Doppler waveform analysis. In VDUS, the B-mode imaging is applied while the lower limb veins are compressed along their extent with a US transducer for presentation of an intraluminal thrombus. A patent vein will exhibit whole vein wall coaptation on compression with the US probe (Figure 1). Absence of compressibility is the most reliable indicator of an existing of thrombus within the studied vein $[9,10]$.

Color flow Doppler is useful for examining residual flow within a thrombosed venous section and for verifying patency of venous segments that are not approachable for compression. The pulsed Doppler spectral waveform in a normal lower limb vein exhibits spontaneous and respirophasic flow pattern (Figure 2). An alteration of this flow pattern might include obstruction proximally to the level of inquiry (Figure 3). Also, respirophasicity could be assessed by distal augmentation maneuvers during spectral Doppler evaluation to further demonstrate patency of the veins. While the distal augmentation maneuver is applied, there should be a sharp "spike" of augmented anterograde venous flow (Figure 2). Blunted 
or abscence of flow augmentation indicates venous obstruction distally $[9,10]$.

The aim of the current study was to evaluate the role of duplex ultrasound (DUS) in the evaluation of deep venous blood flow in fractured lower extremities, to rule out DVT prior to orthopedic surgery.

\section{Material and methods}

\section{Study population}

Between March 2015 and February 2017, a total of 58 patients ( 42 males and 16 females), aged ranged between 18 and 86 years (mean age of $51.5 \pm 19.5$ years) who presented with fractured lower extremities were enrolled in this prospective study. Patients were thoroughly evaluated before surgery with respect to medical history, fracture pattern, associated injuries, comorbid conditions, and VDUS findings. Informed consent was obtained from all patients, and the study was reviewed and approved by an institutional review board.

\section{Ultrasound examination protocol}

Immediately upon patient admission to the ultrasound (US) department, the affected limb was assessed for DVT by VDUS using a Sonoline G 60S US imaging system; Siemens - Germany, equipped with a high frequency $(7-10 \mathrm{MHz})$ linear probe. Gray-scale images and color and spectral Doppler waveforms were recorded without and with compression and from the long axis, respectively using UP-D898MD digital, black and-white A6 printer; Sony and UPP-110S high density standard US thermal paper roll $(110 \mathrm{~mm} \times 20 \mathrm{~m})$. Distal augmentation maneuvers were performed with manual calf compression while investigating the common femoral vein (CFV), superficial femoral vein (SFV), and popliteal vein (POPV) segments. Foot compression was used to evaluate the posterior tibial veins (PTVs). US examination was considered to be negative if there was a normal blood flow in CFV, SFV, POPV, and PTVs, with the vessel lumen fully compressible and completely filled with color. DVT was diagnosed if the vessel wall was not compressed. Because color Doppler ultrasound (CDUS) is operator dependent, all scans were performed by an experienced musculoskeletal US sonologist [11].

US scanning was conducted with the patient in the supine position with the head raised from 15 to $30 \mathrm{de}$ grees and the examination table tilted by 5 to 10 degrees (reverse Trendelenburg tilt). The investigated leg was outwardly rotated at the hip with the knee slightly. In the short axis, starting at CFV and advancing into the distal external iliac vein (EIV), the transducer was moved moderately inferiorly to completely scan CFV and SFV throughout the thigh and to scan POPV from a posterior approach throughout the popliteal fossa. From a poster-

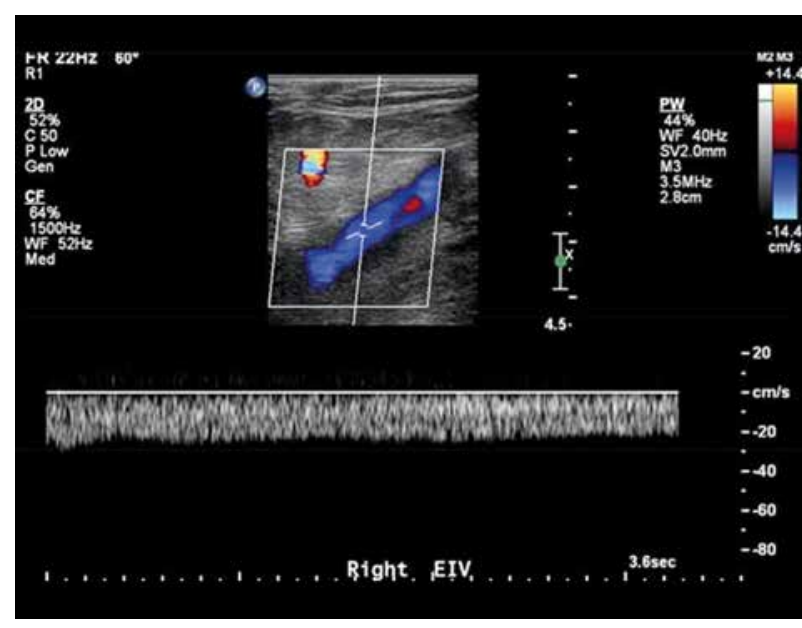

Figure 3. Spectral Doppler waveform analysis of the external iliac vein. The monophasic venous flow, indicates venous obstruction proximally [9]

omedial access in the lower extremity, PTVs were evaluated. Probe compression was applied at 1 to $2 \mathrm{~cm}$ intervals for all vein sections, with each section evaluated for complete compressibility and for the presence of any intraluminal echoes suggestive of thrombus. Longitudinal inspections were applied to assure the presence of intraluminal echoes seen on short axis imaging and to obtain Doppler spectral waveforms of venous hemodynamics in CFV, SFV, POPV, and PTVs. These Doppler waveforms were studied for the presence of spontaneous flow, respiratory phasicity, and augmented flow in response to manual distal limb compression [12]. The patients who were diagnosed with DVT of a CFV, SVF, and/or POPV received warfarin and dalteparin for three months. The international normalized ratio (INR) was used to monitor warfarin treatment. Once the INR was greater than 2 , dalteparin was stopped and warfarin continued.

\section{Statistical analysis}

Study results were initially summarized as a means \pm standard deviations (SD) tables and on graphs. The analysis was performed with the Statistical Package for the Social Sciences (SPSS) version 20 for Windows; Microsoft. Statistical diagnostic testing was used to detect sensitivity and specificity of the absence of phasicity and compressibility in diagnosing DVT in patients with fractured lower extremities.

\section{Results}

In this study, fractures of the lower extremities were classified according to the site of trauma, complexity (simple or compound fracture), and persistent wound either in the peri-acetabular area, femur, knee joint, tibia, and fibula bones. We found single closed fractures in 36 patients (62.1\%), single opened fractures in 9 patients (15.5\%), multiple closed fractures in 10 patients (17.2\%), and multiple 
Table 1. Deep venous thrombosis incidence according to fracture site, BMI, chronic diseases, and smoking status

\begin{tabular}{|c|c|c|c|c|}
\hline Variables & \multicolumn{4}{|c|}{$(n ; \%)$} \\
\hline Fracture classification & Single closed $(36,62.1 \%)$ & Single opened $(9,15.5 \%)$ & Multiple closed $(10,17.2 \%)$ & Multiple opened $(3,5.2 \%)$ \\
\hline $\begin{array}{l}\text { DVT incidence vs. fracture } \\
\text { classification }\end{array}$ & $(11,68.8 \%)$ & $(2,12.5 \%)$ & $(2,12.5 \%)$ & $(1,6.2 \%)$ \\
\hline BMI & \multicolumn{2}{|c|}{$<25 \mathrm{~kg} / \mathrm{m}^{2}(39,67.2 \%)$} & \multicolumn{2}{|c|}{$>25 \mathrm{~kg} / \mathrm{m}^{2}(19,32.8 \%)$} \\
\hline DVT incidence vs. BMI & \multicolumn{2}{|c|}{$(7,43.8 \%)$} & \multicolumn{2}{|c|}{$(9,56.3 \%)$} \\
\hline Chronic diseases & DM $(11,19 \%)$ & \multicolumn{2}{|c|}{$\operatorname{HTN}(6,10.3 \%)$} & DM \& HTN $(1,1.7 \%)$ \\
\hline $\begin{array}{l}\text { DVT incidence versus chronic } \\
\text { diseases }\end{array}$ & $(5,31.3 \%)$ & \multicolumn{2}{|c|}{$(3,18.8 \%)$} & $(0,0 \%)$ \\
\hline Smoking status & \multicolumn{2}{|c|}{ Smokers $(7 ; 12.1 \%)$} & \multicolumn{2}{|c|}{ Nonsmokers (51; 87.9\%) } \\
\hline $\begin{array}{l}\text { DVT incidence versus smoking } \\
\text { status }\end{array}$ & \multicolumn{2}{|c|}{$(2 ; 12.5 \%)$} & \multicolumn{2}{|c|}{$(14 ; 87.5 \%)$} \\
\hline Contraceptive pills & \multicolumn{2}{|c|}{$\begin{array}{l}\text { Females using contraceptive pills } \\
\qquad(1,1.7 \%)\end{array}$} & \multicolumn{2}{|c|}{$\begin{array}{l}\text { Females not using contraceptive pills } \\
\qquad(15,25.9 \%)\end{array}$} \\
\hline $\begin{array}{l}\text { DVT incidence versus } \\
\text { contraceptive pill use }\end{array}$ & \multicolumn{2}{|c|}{$(1,6.25 \%)$} & \multicolumn{2}{|c|}{$(4,25 \%)$} \\
\hline
\end{tabular}

DVT - deep venous thrombosis, BMI - body mass index, DM - diabetes mellitus, HTN - hypertension

opened fractures in 3 patients (5.2\%). A total of 39 (67.2\%) patients had the body mass index $(\mathrm{BMI})<25 \mathrm{~kg} / \mathrm{m}^{2}$, while $19(32.8 \%)$ patients had the BMI $>25 \mathrm{~kg} / \mathrm{m}^{2}$. There was $11(19 \%)$ patients with diabetes mellitus (DM), 6 (10.3\%) with hypertension (HTN), and a combination of DM and HTN was seen in 1 patient (1.7\%). It should be noted that DVT was present in $5(31.3 \%)$ patients with DM, and 3 patients (18.8\%) with HTN. A total of 7 (43.8\%) patients with DVT had BMI $<25 \mathrm{~kg} / \mathrm{m}^{2}$, and 9 patients (56.3\%) with DVT had BMI $>25 \mathrm{~kg} / \mathrm{m}^{2}$. Two patients (12.5\%) with DVT were smokers and continued to smoke during the course of the study. According to the classification of fracture depending on the site of trauma, DVT was found in $11(68.8 \%)$ patients with single closed fractures, $2(12.5 \%)$ patients with single opened fractures, $2(12.5 \%)$ patients with multiple closed fractures, in $1(6.2 \%)$ patient with multiple closed fractures,

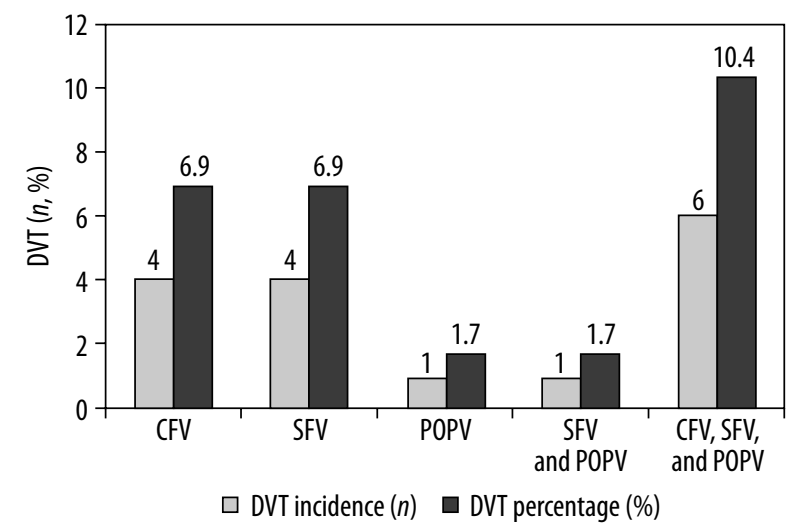

CFV - common femoral vein, SFV - superficial femoral vein, POPV - popliteal vein

Figure 4. Lower extremity deep venous thrombosis sites (number, and percentage of incidence) and 3 (5.2\%) patients with multiple opened fractures. Only $1(1.7 \%)$ female patient used contraceptive pills and had DVT. The rest of female patients $(15,25.9 \%)$ did not use contraceptive pills, but in 4 of them (25\%) we detected DVT (Table 1). In addition to DVT, other US findings were as follows: Baker's cyst $(1,1.7 \%)$, lymphadenopathy $(16,27.6 \%)$, and intramuscular hematomas $(2,3.5 \%)$.

VDUS showed that $16(27.6 \%)$ patients with fractured lower extremities had DVT. In 4 (6.9\%) cases, DVT involved either CFV or SFV. We observed DVT in single patient (1.7\%) in POPV, SFV and POPV. Six (10.3\%) patients had DVT in CFV, SFV, and POPV (Figure 4).

Absence of compressibility $(4,6.9 \%)$ and phasicity $(4,6.9 \%)$ was detected in $4(6.9 \%)$ cases of DVT involving CFV. Absence of compressibility $(4,6.9 \%)$ was noted also in $4(6.9 \%)$ cases of DVT in SFV, but absence of phasicity

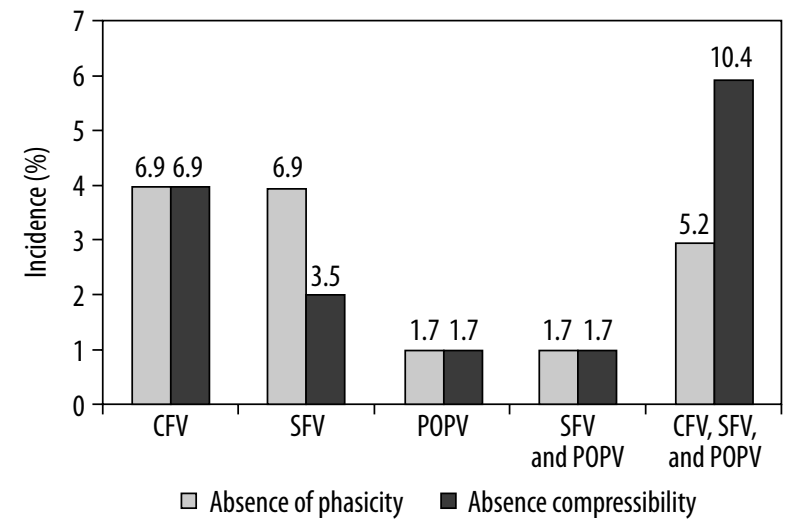

CFV - common femoral vein, SFV - superficial femoral vein, POPV - popliteal vein

Figure 5. Absence of compressibility and phasicity (\%) in deep venous thrombosis sites 
Table 2. Performance of absence of compressibility and phasicity in the detection of deep venous thrombosis in fractured lower extremities

\begin{tabular}{|c|c|c|c|c|c|}
\hline $\begin{array}{l}\text { Absence of compressibility } \\
\text { in detection of DVT in fractured } \\
\text { lower extremities }\end{array}$ & $\begin{array}{l}\text { Number of } \\
\text { cases }(n)\end{array}$ & \multicolumn{2}{|c|}{$\begin{array}{l}\text { Absence of phasicity in detection of DVT } \\
\text { in fractured lower extremities }\end{array}$} & \multicolumn{2}{|c|}{ Number of cases ( $n$ ) } \\
\hline True positive & 13 & \multicolumn{2}{|c|}{ True positive } & \multicolumn{2}{|c|}{14} \\
\hline True negative & 45 & \multicolumn{2}{|c|}{ True negative } & \multicolumn{2}{|c|}{44} \\
\hline False positive & 0 & \multicolumn{2}{|c|}{ False positive } & \multicolumn{2}{|c|}{0} \\
\hline False negative & 3 & \multicolumn{2}{|c|}{ False negative } & \multicolumn{2}{|c|}{2} \\
\hline $\begin{array}{l}\text { Performance of absence of } \\
\text { compressibility in detection of } \\
\text { DVT in fractured lower extremities }\end{array}$ & Value & $95 \% \mathrm{Cl}$ & $\begin{array}{l}\text { Performance of absence of } \\
\text { phasicity in detection of DVT } \\
\text { in fractured lower extremities }\end{array}$ & Value & $95 \% \mathrm{Cl}$ \\
\hline Sensitivity (\%) & 81.25 & $54.35-95.95$ & Sensitivity (\%) & 87.50 & $61.65-98.45$ \\
\hline Specificity (\%) & 100.00 & $92.13-100.00$ & Specificity (\%) & 100.00 & $91.96-100.00$ \\
\hline Negative likelihood ratio & 0.19 & $0.07-0.52$ & Negative likelihood ratio & 0.12 & $0.03-0.46$ \\
\hline $\begin{array}{l}\text { DVT prevalence in fractured } \\
\text { lower extremities }(\%)\end{array}$ & 26.23 & $15.80-39.07$ & $\begin{array}{l}\text { DVT prevalence in fractured } \\
\text { lower extremities (\%) }\end{array}$ & 26.67 & $16.07-39.66$ \\
\hline PPV (\%) & 100.00 & - & PPV (\%) & 100 & - \\
\hline NPV (\%) & 93.75 & $84.40-97.65$ & NPV (\%) & 95.65 & $85.75-98.77$ \\
\hline
\end{tabular}

DVT - deep venous thrombosis, PPV - positive predictive value, NPV - negative predictive value

was noted only in $2(3.5 \%)$ of the affected patients. Absence of compressibility $(1,1.7 \%)$ and phasicity $(1,1.7 \%)$ was found in single (1.7\%) DVT cases that affected either POPV or SFV or POPV. In 6 (10.4\%) DVT cases in CFV, SFV, and POPV, absence of both compressibility and phasicity was noted in $3(5.2 \%)$ and $6(10.4 \%)$ cases, respectively (Figure 5).

Diagnostic testing revealed sensitivity of $81.25 \%$ (95\% CI: 54.35-95.95), and specificity of $100 \%$ (95\% CI: 92.13-100) of absence of compressibility in the detection of DVT in fractured lower extremities.

Furthermore, we found positive predictive value (PPV) of $100 \%$ and negative predictive value (NPV) of 93.75\% (Table 2). In addition, absence of phasicity had sensitivity of $87.50 \%$ (95\% CI: 61.65-98.45) and specificity of $100 \%$ (95\% CI: 91.96-100) in diagnosis of DVT in fractured lower extremities. PPV and NPV in this case were $100 \%$ and $95.65 \%$, respectively (Table 2 ).

The prevalence of DVT in fractured lower extremities was $26.23 \%$ (95\% CI: $15.80-39.07$ ) or $26.67 \%$ (95\% CI: 16.07-39.66), when using absence of compressibility and phasicity to confirm the presence of intraluminal thrombus, respectively (Table 2).

\section{Discussion}

In the current study, fractures of the lower extremities were classified according to the site of trauma, complexity, and wound location either in the peri-acetabular area, femur, knee joint, tibia, fibula, or multiple sites. We found single closed fractures $(36,62.1 \%)$, single opened fractures $(9,15.5 \%)$, multiple closed fractures $(10,17.2 \%)$, and multiple opened fractures (3, 5.2\%). According to the site of trauma, we found DVT in single closed fractures $(11,68.8 \%)$, single opened fractures $(2,12.5 \%)$, multiple closed fractures $(2,12.5 \%)$, and multiple opened fractures (1, 6.2\%) (Table 1). Such findings could be compared to several studies, such as that by as Wenzl et al. [13], in which the authors prospectively analyzed dalteparin, a low molecular weight heparin (LMWH), in patients with orthopedic trauma. With dalteparin use, about $2.4 \%$ (10 of 411) of patients with a femoral fracture developed DVT. Goel et al. [14] examined the occurrence of DVT following unilateral fractures below the knee that required venography in connection with surgery. The patients were categorized into two groups and treated with LMWH for thromboembolic prophylaxis or with placebo. Venography was conducted two weeks after surgery. A higher incidence of tibia plateau fractures was associated with DVT after surgery. Thromboembolic events occured two to four weeks after surgery, and patients with ankle fractures may be at a high risk during this period. Lapidus et al. [15] analyzed DVT in patients with ankle fractures that required surgery. All patients were treated with heparin within the first postoperative week. Subsequently, only $50 \%$ of patients received DVT prophylaxis. Based on phlebography, DVT was found in $21 \%$ of patients, while CDUS confirmed DVT in $31 \%$ of patients. Lapidus et al. [16] studied the prophylactic effect of LMWH (dalteparin) compared to placebo. After using LMWH (dalteparin), 21\% of patients developed DVT, in contrast to $28 \%$ of pateints without prophylaxis. The presence of DVT after an isolated lower limb trauma seems to range between $5 \%$ and $86 \%$, while femur fractures bear the 
highest risk of DVT, followed by tibia plateau fractures. Tibial shaft fractures and ankle fractures seem to bear a similar risk [17].

Traumatized veins may undergo post-traumatic thrombosis, and this may change the normal characteristics of these vessels. The presence of hematoma at the site of major veins without visualization of the vein is a robust indicator of whole vein rupture. In trauma patients, vein thrombosis develops minutes or hours after trauma. For the diagnosis of DVT, one can apply compression techniques and CDUS $[18,19]$. In normal veins, blood flow is uninterrupted and spontaneous at rest, and the velocity changes with respiration, rising on inhalation and falling on exhalation. Active compression of the distal parts of the legs causes an increase or augmentation of the velocity. In the case of partial thrombosis, blood flow may be normal or abnormal, without response to respiration. In complete obstructive thrombosis, there will be an entire absence of blood flow on DUS investigation [7].

In the current of study, VDUS showed that some patients with fractures lower extremities had DVT $(16,27.6 \%)$ (Figure 4$)$. The absence of compressibility $(4,6.9 \%)$ and phasicity $(4,6.9 \%)$ was detected in DVT invloving CFV (4, 6.9\%), Absence of compressibility $(4,6.9 \%)$ was noted in DVT involving SFV $(4,6.9 \%)$, but the absence of phasicity was noted only in 2 (3.5\%) of the affected patients. An equal incidence of absence of compressibility and phasicity was found in DVT that affected solely POPV (1, 1.7\%), SFV (1, 1.7\%), or POPV $(1,1.7 \%)$. As regards the cases in which DVT involved CFV, SFV, and POPV $(6,10.4 \%)$, absence of compressibility and phasicity was found in $3(5.2 \%)$ and $6(10.4 \%)$ patients, respectively (Figure 5).

Color flow Doppler (CFD) is currently considered as the first-line examination for evaluation of vascular injuries, with sensitivity and specificity of $89-100 \%$ relative to venography. Moreover, CFD has an accuracy of $95 \%$ to $98 \%$ in detecting lower extremity DVT $[8,20]$. In the current study, diagnostic testing revealed sensitivity of $81.25 \%$ and specificity of $100 \%$ of absence of compressibility in the detection of DVT in fractured lower extremities. Furthermore, positive predictive value (PPV) of $100 \%$ and negative predictive value (NPV) of $93.75 \%$ were found (Table 2). In addition, absence of phasicity in the diagnosis of DVT in fractured lower extremity has sensitivity of $87.50 \%$ and specificity of $100 \%$. PPV and NPV in this case were $100 \%$ and $95.65 \%$, respectively (Table 2 ).

Obesity, large hematomas, emphysema, and large uncovered wounds of the skin can hinder US examination performed in order to rule out trauma-related vascular injuries [8,21]. Further limitations are due to operator dependence and duration of examination, which may be inappropriate in an acute setting. Despite these limitations, venous US can help diagnose other pathologies such as Baker's cysts, superficial or intramuscular hematomas, lymphadenopathy, femoral aneurysm, superficial thrombophlebitis, and absceses. Moreover, there is no exposure to radiation [22]. In the current study, we diagnosed Baker's cyst (1,1.7\%), lymphadenopathy $(16,27.6 \%)$, and intramuscular hematomas $(2,3.5 \%)$.

\section{Conclusions}

In conclusion, we evaluated the role of DUS in the detection of deep venous blood flow in fractured lower extremities to rule out DVT prior to orthopedic surgery. The incidence of DVT after orthopedic trauma was high $(16,27.6 \%)$ and appears to be associated with fractures of the peri-acetabular area, femur, knee joint, tibia, and fibula. VDUS with gray-scale imaging with compression maneuvers should be the first-line imaging modality for suspected lower extremity DVT. Loss of phasicity and compressibility in a venous segment, often with accompanying Doppler abnormalities, indicates DVT with a high degree of accuracy, and no additional examination is required to initiate management.

\section{Acknowledgements}

The authors would like to thank the staff of the ultrasound department of the Ribat University Hospital for their cooperation and support during data collection.

\section{Conflicts of interest}

The authors report no conflict of interest.
1. Oger E. Incidence of venous thromboembolism: A community-based study in Western France. EPI-GETBP Study Group. Groupe d'Etude de la Thrombose de Bretagne Occidentale. Thromb Haemost 2000; 83: 657-660.

2. Murphy RF, Naqvi M, Miller PE, et al. Pediatric orthopaedic lower extremity trauma and venous thromboembolism. J Child Orthop 2015; 9: 381-384.
3. Kitagawa K, Sakoda S. Mechanism underlying thrombus formation in cerebral infarction. Rinsho Shinkeigaku 2009; 49: 798-800.

4. Hak DJ. Prevention of venous thromboembolism in trauma and long bone fractures. Curr Opin Pulm Med 2001; 7: 338-343.

5. Knudson MM, Ikossi DG, Khaw L, et al. Thromboembolism after trauma: An analysis of 1602 episodes from the American College of Surgeons National Trauma Data Bank. Ann Surg 2004; 240: 490-498. 
6. Rozycki GS, Tremblay LN, Feliciano DV, et al. Blunt vascular trauma in the extremity: diagnosis, management, and outcome. J Trauma 2003; 55: 814-824.

7. Montorfano MA, Pla F, Vera L, et al. Point-of-care ultrasound and Doppler ultrasound evaluation of vascular injuries in penetrating and blunt trauma. Crit Ultrasound J 2017; 9: 5.

8. Gaitini D, Razi NB, Ghersin E, et al. Sonographic evaluation of vascular injuries. J Ultrasound Med 2008; 27: 95-107.

9. Gornik HL, Sharma AM. Duplex ultrasound in the diagnosis of lowerextremity deep venous thrombosis. Circulation 2014; 129: 917-921.

10. Kassaï B, Boissel JP, Cucherat M, et al. A systematic review of the accuracy of ultrasound in the diagnosis of deep venous thrombosis in asymptomatic patients. Thromb Haemost 2004; 91: 655-666.

11. Patil S, Gandhi J, Curzon I, et al. Incidence of deep-vein thrombosis in patients with fractures of the ankle treated in a plaster cast. J Bone Joint Surg Br 2007; 89: 1340-1343.

12. Bendick PJ, Glover JL, Brown OW, et al. Serial duplex ultrasound examinations for deep vein thrombosis in patients with suspected pulmonary embolism. J Vasc Surg 1996; 24: 732-737.

13. Wenzl ME, Hasse W, Seide K, et al. Prevention of thromboembolism with low-molecular-weight heparin in orthopedic surgery: A 5-year experience. Clin Appl Thromb Hemost 2004; 10: 1-4.

14. Goel DP, Buckley R, deVries G, et al. Prophylaxis of deep-vein thrombosis in fractures below the knee: A prospective randomised controlled trial. J Bone Joint Surg Br 2009; 91: 388-394.
15. Lapidus L, de Bri E, Ponzer S, et al. High sensitivity with color duplex sonography in thrombosis screening after ankle fracture surgery. J Thromb Haemost 2006; 4: 807-812.

16. Lapidus LJ, Ponzer S, Elvin A, et al. Prolonged thromboprophylaxis with Dalteparin during immobilization after ankle fracture surgery: A randomized placebo-controlled, double-blind study. Acta Orthop 2007; 78: 528-535.

17. Decker S, Weaver MJ. Deep venous thrombosis following different isolated lower extremity fractures: What is known about prevalences, locations, risk factors and prophylaxis? Eur J Trauma Emerg Surg 2013; 39: 591-598.

18. Burnside PR, Brown MD, Kline JA. Systematic review of emergency physician-performed ultrasonography for lower-extremity deep vein thrombosis. Acad Emerg Med 2008; 15: 493-498.

19. Bernardi E, Camporese G, Büller HR, et al. Serial 2-point ultrasonography plus D-dimer vs. whole-leg color-coded Doppler ultrasonography for diagnosing suspected symptomatic deep vein thrombosis: A randomized controlled trial. JAMA 2008; 300: 1653-1659.

20. Hammers LW, Cohn SM, Brown JM, et al. Doppler color flow imaging surveillance of deep vein thrombosis in high-risk trauma patients. J Ultrasound Med 1996; 15: 19-24.

21. Schoder M, Prokof M, Lammer J. Traumatic injuries: Imaging and intervention of large arterial trauma. Eur Radiol 2002; 12: 1617-1631.

22. Kesieme E, Kesieme C, Jebbin N, et al. Deep vein thrombosis: A clinical review. J Blood Med 2011; 2: 59-69. 